\title{
Unusual Case of Pyoderma Gangrenosum and Bilateral Ear Changes Secondary to the Use of Cocaine with Levamisole in a Patient with Systemic Lupus Erythematodes
}

\author{
Manov A*, Dhanabalsamy $\mathbf{N}$ and Hapuarachchi $\mathbf{M}$ \\ UNTHSC School of Medicine, City of Fort Worth Hospital, USA
}

\section{Case Report}

Volume 3 Issue 2

Received Date: April 01, 2020

Published Date: April 17, 2020

DOI: $10.23880 /$ abca-16000116

with raised edges which were well demarcated and painful.

\section{Introduction}

Levamisole has been considered an adulterant in cocaine and increasingly reported as the cause for many of the complications noted in cocaine users [1-3]. The main reason for intentionally adding this compound to street cocaine has been due to its effect to potentiate the sympathomimetic effects of cocaine. The exact mechanism by which this agent causes complications is unclear. We present a unique case of a cocaine user who presented with acute non-healing skin lesions, ear changes, inflammatory monoarthritis and other systemic signs with subsequent workup leading to the diagnosis.

\section{Case Presentation}

A 55-year-old African American female with past medical history significant for Systemic Lupus Erythematous, Herpes simplex infection and recent history of right nonhealing inguinal ulceration presented with increasing pain in the right non-healing inguinal ulceration site with some yellowish discharge. The size of the ulceration has progressively gotten worse. The patient also reported 1-2 day history of necrotic lesions on her bilateral forearms, left lower extremity below the knee and swelling, pain and necrotic lesion on her tongue. She was a current every day smoker, denied alcohol and use of recreational drugs. Right inguinal big ulceration was with pustular lesions and also serosanguinous drainage. It was very painful to touch. Discoloration and swelling of the cartilage of the both ears sparing the pinna was observed. Edema in the left ankle with warmth, erythema was observed with pain on active and passive motions suggestive of monoarthritis of the left ankle which was proved to be inflammatory and noninfectious on diagnostic tap of the left ankle. Also a round necrotic lesion on the left lower extremity below the knee was observed
Labs on admission showed low WBC count, neutropenia, microcytic anemia and elevated procalcitonin level, ESR, CRP level were also found. Immunological workup yielded positive ANA, c-ANCA titer 1:640, elevated P ANCA or MPOANCA, decreased C3, C4 and CH50 levels, elevated HSV 1\&2 IgG Ab, elevated HCV RNA, Hepatitis C 1 A genotype, negative cryoglobulins, elevated single and double stranded DNA AB and High CMV IgG. Urine drug test was positive for cocaine.

Based on the presenting symptoms of inflammation, suspecting superimposed bacterial infection, IV broad spectrum antibiotics and antiviral drugs were begun without improvement of the skin and ear lesions and left ankle arthritis. On further questioning, the patient admitted to using cocaine with levamisole and reported recurrent similar lesions for 3-years only after the usage of inhaled cocaine with levamisole.

Surgical biopsy of the right inguinal lesion and left lower extremity lesion was performed. Pathology with light microscopy and direct immunofluorescence results were supportive of the diagnosis of pyoderma gangrenosum and leukoclastic vasculitis.

The sample grew also Bacteroides fragilis, E cloacae, E faecalis, $S$ epidermidis which we believed represented secondary bacterial infection and continue the antibiotics but stopped the antiviral drugs.

The final Diagnosis of Pyoderma gangrenosum and vasculitis secondary to Levamisole-cocaine was made based on the clinical presentation and association with their usage of the skin and ear findings. Also the inflammatory left ankle arthritis we believe was due to $\mathrm{L}$. 
The patient was started on Steroids with significant improvement of the skin lesions in the right inguinal area, left lower extremity, necrotic lesions on both upper extremities and tongue. The ear lesions completely resolved after the start of the steroids as well as the monoarthritis of the left ankle. Also the inflammatory left ankle arthritis we believe was due to cocaine-levamisole. Few cases are described in the literature.

The patient was discharged on tapering doses of steroids and per oral antibiotics.

\section{Discussion}

Initially described in 1978 in a patient treated for rheumatoid arthritis, levamisole-induced skin findings are becoming more evident in clinical practice these days. This is because, in the USA, it is now estimated that about $70 \%$ of the cocaine is adulterated with levamisole as it not only adds volume to the drug but is also believed to potentiate psychotropic properties of the cocaine. Pyoderma gangrenosum /PG/ is one of the neutrophilic dermatoses which are associated with SLE, Rheumatoid arthritis, Hepatitis C, Inflammatory bowel diseases, cryoglobulinemia etc. as well as medications like isotretinoin, sunitinib, propylthiouracil etc. Other neutrophilic dermatoses are Sweet syndrome, Behcet's disease and neutrophilic urticarial. Recently in the medical literature there are few reports of association of PG with cocaine- levamisole.

In PG initially there is neutrophil infiltration (with or without lymphocytes), which often occurs diffusely in the dermis and involves follicular structures. There are varying degrees of vascular damage, but usually without fibrinoid necrosis. Focal vasculitis is observed in fully developed lesions, which is apparently a secondary phenomenon. The infiltrate tends to be located in deeper levels of the dermis. A mixed inflammatory process and areas of necrosis are observed in fully developed lesions. There is a wide clinical variability, but the morphology of $P G$ is the following: a) pustular b) ulcerated c) vesico-bullous, d) verrucous vegetating. In general, there is predominance of one of the clinical forms, which can coexist in the same patient.

In our patient, the pustular and ulcerated form of PG coexisted. The bacterial infections were secondary to the underlying PG. There was no response to the initial antibacterial antibiotic and antiviral treatment of the lesions, but significant response to steroids.

Pathological diagnosis confirmed PG. The cause of it might have been SLE flare, or Hepatitis $\mathrm{C}$ but the previous as well as current flare of the PG was always associated with the use of inhaled cocaine with Levamisole which made us think that this was the most likely cause of the PG [4].

Additionally, the most pathognomonic lesions of cocaine- levamisole syndrome are those on the ears which our patient had. This is why we believe when we followed the clinical, laboratory, histological and treatment picture that the cause of the PG and ear changes was the cocainelevamisole [5-8]. The patient did not fulfill the criteria for Relapsing Polychondritis [9].

The cocaine-levamisole related syndrome comprises a set of immunological abnormalities, out of which, ANCA positivity is the most important one. Our patient was ANCA positive also.

Regarding pathological findings in cocaine adulterated with levamisole syndrome, this can range from the classic finding of leukocytoclastic vasculitis of small vessels to occlusive vascular disease without true vasculitis.

Although the mechanism of how tainted cocaine causes characteristically clinical and immunological findings is not well known, there are suggested theories. The main theory nowadays relates the direct cytotoxic effect of levamisole to the cells, especially neutrophils, is of consideration. It is this direct effect due to which case reports of the association between pyoderma gangrenosum and the use of adulterated cocaine are emerging as pyoderma gangrenosum's pathophysiological basis is endothelial damage. Furthermore, ANCA translocation and increased recruitment of neutrophils all contribute to the damage of the vessel by oxidative reaction and degranulation. Also, cocaine-levamisole might act as immunomodulator and cause immune attack of the cartilaginous portion of the ears as in our patient.

Also the inflammatory left ankle arthritis we believe was due to cocaine-levamisole. Few cases are described in the literature [10].

\section{Conclusion}

In conclusion, we think that cocaine- levamisole syndrome is increasing and the clinicians should be aware of the clinical manifestation of the syndrome- skin findings suggestive of vasculitis, or non-vasculitis occlusive disease of the small vessels, neutrophil dermatoses like PG and inflammatory ear changes among others and not always attribute those changes to Rheumatological or Infectious disorders like Systemic Lupus erythematosus/SLE/, Rheumatoid arthritis/RA/, Hepatitis C etc. Besides the thorough work up which we did the clinicians should follow the clinical presentations and temporary association of the symptoms with the disease flare or the drug use like in our 


\section{Annals of Bioethics \& Clinical Applications}

patient when the skin and ear changes always happened with the use of cocaine- levamisole.

\section{References}

1. Chang A, Osterloh J, Thomas J (2010) Levamisole: a dangerous new cocaine adulterant. Clin Pharmacol Ther 88(3): 408-411.

2. Hays PA (2012) Technical Note Identification of Levamisole Impurities Found in Illicit Cocaine Exhibits. Microgram, pp: 82.

3. Baptiste GG, Alexopoulos AS, Masud T, Bonsall JM (2015) Systemic levamisole-induced vasculitis in a cocaine user without cutaneous findings: a consideration in diagnosis. Case reports 2015: 3 .

4. Nolan AL, Jen KY (2015) Pathologic manifestations of levamisole-adulterated cocaine exposure. Diagn Pathol 10(1): 48.

5. Ruiz Villaverde R, Sanchez Cano D (2016) Multiple pyoderma gangrenosum ulcers associated with cocaine abuse. Sultan Qaboos Univ Med J 16(4): 527-528.

6. Garg S, Moesch J, Conn D (2016) Levamisole induced Vasculopathy: A Case of Extensive Necrosis after Reexposure to Levamisole Tainted Cocaine. Int J Clin Med Images 3(3): 1-2.

7. Jeong HS, Layher H, Cao L, Vandergriff T, Dominguez AR (2016) Pyoderma gangrenosum (PG) associated with levamisole-adulterated cocaine: clinical, serologic, and histopathologic findings in a cohort of patients. J Am Acad Dermatol 74(5): 892-898.

8. Abdul Karim R, Ryan C, Rangel C, Emmett M (2013) Levamisole-induced vasculitis. Proc (Baylor Univ Med Cent) 26(2): 163-165.

9. Pearson CM, Kline HM, Newcomer VD (1960) Relapsing Polychondritis. N Engl J Med 263: 51-58.

10. Emil N, Cisneros DR, Penmetsa S, Duchesne JH, Sibbitt WL, et al. (2020) Atypical Chronic inflammatory ANCApositive Deforming Arthritis after Cocaine-Levamisole Exposure. J Clin Rheumatol 26(1): 24-32. 